\title{
Valoración de una empresa de agua potable mediante opciones reales
}

\section{RESUMEN}

Valorar una empresa representa un desafío mayor ya que es necesario incorporar los diversos factores de riesgo, para determinar su valor "justo". La metodología de opciones reales muestra un precio por acción de la empresa Aguas Andinas S.A. en torno a los $\$ 473$ (U\$ 0,68) por acción, resultado superior a los precios históricos de la empresa. Una revisión de los informes del mercado revela confianza en esta empresa, basándose en su plan de inversiones y en las proyecciones de su negocio. Los resultados muestran que la metodología de opciones reales genera un valor adicional, debido principalmente al impacto positivo que tiene el riesgo sobre el valor de la acción y a la variabilidad de los ingresos. La metodología de opciones reales requiere mayor investigación, para una resolución definitiva.

Palabras clave: valoración, empresa, sanitaria, opciones, reales

VALUATION DRINKING WATER FIRMS THROUGH REAL OPTIONS

\section{ABSTRACT}

Valuation a company represents a major challenge since it is necessary to incorporate various risk factors to determine their "fair" value. The real options method show stock price for Aguas Andinas company, around $\$ 473$ (U\$ 0.68) per share, higher than the historical result of the action. A review of market reports reveals confidence in this company, based on its investment plan and business projections. The results show that real options methodology generates additional value, mainly due to the positive impact of the risk on the value of the action and the variability of income. The methodology of real options requires further investigation, for a final decision.

Keywords: valuation, firm, real option

\section{INTRODUCCIÓN}

Valorar una empresa siempre ha significado un desafío, ya que entran en juego múltiples factores internos y externos, tangibles e intangibles, económicos, sociales, productivos, legales, de mercado, los que de no ser considerados o, si por otro lado, son erróneamente definidos, arrojarán resultados lejanos a la realidad, es decir, una cifra que no represente el real valor de la empresa. Esto último es indispensable en situaciones en que la toma de decisiones involucra procesos tales como fusiones, adquisición o venta de una empresa, capitalizaciones, obtención de líneas de financiamiento, compraventa de acciones, etc.

El artículo que se presenta a continuación trata sobre la valoración de una empresa de agua potable, aplicando análisis del riesgo y su importancia en las valoraciones de los activos financieros, para tal efecto se explica el modelo de negocios y se analiza el mercado y la industria para aplicar la metodología de opciones reales.

La razón de aplicar este estudio en el sector sanitario se basa en diversos factores. El primero es que Chile es visto a menudo como un ejemplo exitoso a nivel mundial en el desarrollo de la industria del tratamiento del agua. Este desarrollo se manifestó en toda su magnitud a partir del proceso de transferencia de la propiedad de las compañías de agua potable a manos privadas (Alegría y Celedón, 2006). Hasta el momento, este sector ha mostrado óptimo desempeño y estabilidad en el tiempo con crecimientos sostenibles.

Además del beneficio social directo de contar con acceso a redes de agua potable derivado del accionar natural de la empresa, se puede comprobar que la actividad de industria sanitaria tiene amplia presencia en todos los hogares del país.

Aguas Andinas S.A. pertenece a una industria regulada con un marco regulatorio estable, conocido y probado. Es la principal empresa sanitaria del país y una de las mayores de América Latina. Entrega servicios bien definidos que explican claramente el precio de acción. Además cotiza en bolsa lo que asegura un acceso claro y expedito a la información.

\footnotetext{
Profesor Departamento Economía y Finanzas, Universidad del Bío Bío, Chile. E-mail: mauricio.g@ubiobio.cl

** Magíster en Dirección de Empresas, Universidad del Bío Bío, Chile.

E-mail: jhenriquezc@minvu.cl
} 
El objetivo de este artículo es realizar una valoración de la empresa a través del modelo tradicional de los flujos de caja descontados, ajustado por riesgos y compararlos con la valoración mediante la valoración de los activos de la empresa, que sugiere la teoría de opciones reales. En la primera sección se revisa en forma resumida y esquemática las metodologías de valoración de empresas, luego se presente el caso de la empresa sanitaria chilena, posteriormente se realiza la valoración de acuerdo a los modelos tradicionales y adicionalmente las opciones reales, para arribar a las conclusiones definitivas.

\section{ANÁLISIS TEÓRICO}

Los métodos de valoración, cualquiera sea su naturaleza, persiguen un mismo objetivo; estimar el valor de una compañía. Los distintos métodos se pueden agrupar según si se basan en el balance de la empresa, si lo hacen en el descuento de flujos o si utilizan los múltiplos de la compañía en forma comparativa. En el mundo de las finanzas, el concepto de riesgo se relaciona con la posibilidad de ocurrencia de un evento. Lo que se traduce en que existe la posibilidad de que los beneficios sean menores a los esperados. El riesgo es producto de la incertidumbre que existe sobre el valor de los activos financieros, ante movimientos adversos de los factores que determinan su precio; a mayor incertidumbre mayor riesgo Markowitz (1952).

Como mencionan Sapag y Sapag (2000) el comportamiento de los flujos de caja es incierto, puesto que no es posible conocer con anticipación cuál de todos los hechos que pueden ocurrir y que tienen efectos en los flujos de caja, ocurrirá efectivamente. Las fuerzas del mercado, entre otras, generan "presiones" sobre las estimaciones las que, con frecuencia, difieren significativamente de las que se anticipan en los planes originales (Gutiérrez y González 2014).

El hecho de no tener la certeza sobre los flujos de caja futuros nos pone en presencia del riesgo o incertidumbre Entenderemos por riesgo a la variabilidad de los flujos de caja reales respecto de los estimados. En cuanto a la incertidumbre, se tiene que esta se hace más importante a medida que transcurre el tiempo ya que las condiciones estipuladas originalmente son afectadas por el desarrollo del medio en que se desenvuelve la empresa. Así se tiene por ejemplo que variables significativas como precio y calidad de las materias primas, el nivel tecnológico de la producción, las remuneraciones, los mercados, los proveedores, la demanda y un sinfín de otras son condicionadas y modificadas sin poder anticipar el efecto final de aquello en el flujo de caja de la compañía.

Figura 1. Valor Potencial de una empresas, considerando opciones

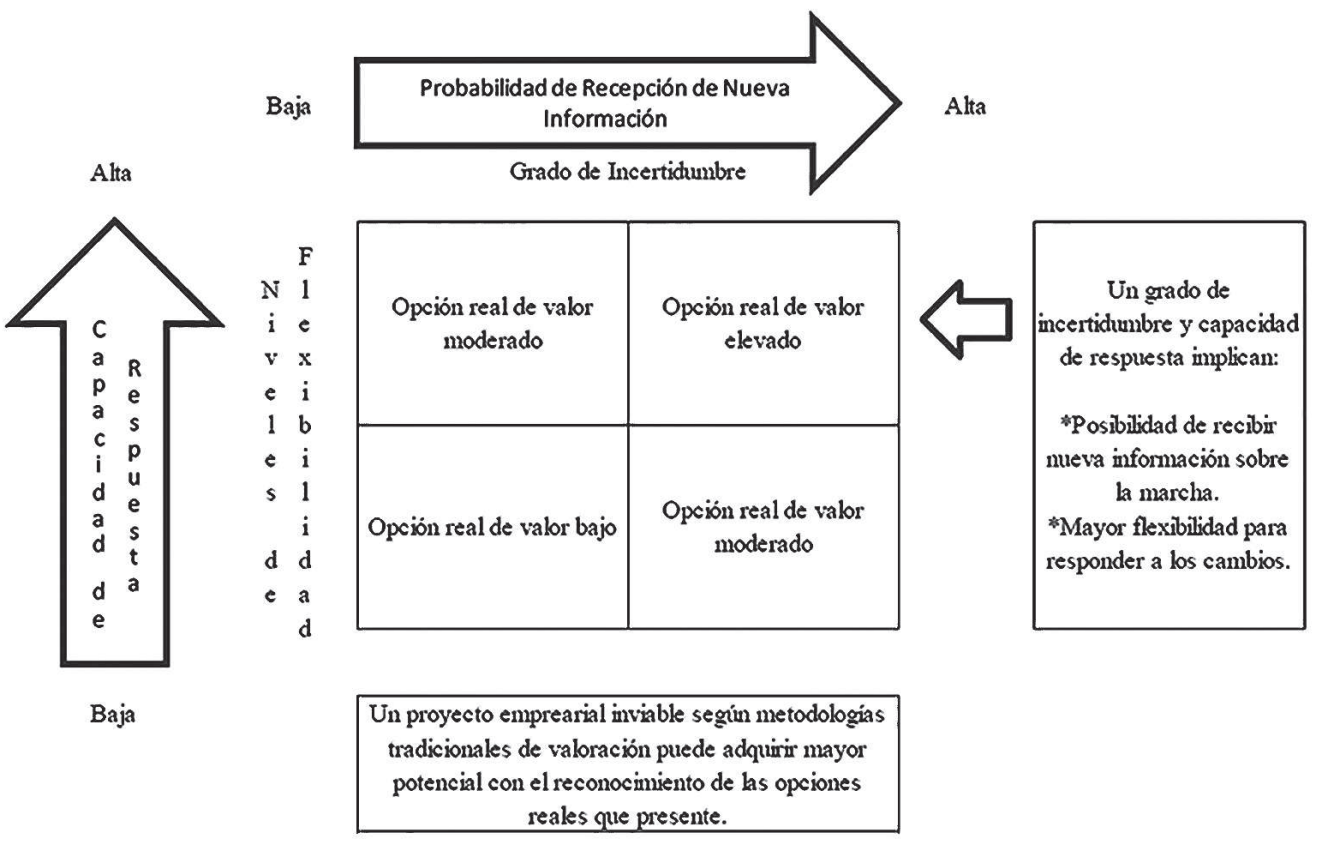

Fuente: Gallardo y Andalaf (2008). Figura utilizada con el consentimiento expreso de los autores Macarena Gallardo y Alejandro Andalaf. 
Adicionalmente se deben agregar eventos que suponen nuevas oportunidades de crecimiento futuro. Así se tiene por ejemplo, la decisión de realizar un proyecto de inversión puede representar la posibilidad de adquirir promesas de desarrollo a futuro, algo que en el momento inicial de evaluar y valorar usando sólo la información que entrega la metodología tradicional no serían considerados. Se dice entonces que este tipo de metodologías tiende a subestimar el valor de las empresas ya que no recogen las nuevas oportunidades que se presentan como fruto de la disponibilidad de nueva información en etapas futuras. De ahí que una metodología más apropiada debiera considerar los datos provenientes de los flujos de caja y también de la propia capacidad futura de la empresa y del mercado de generar nueva información.

Como indican a su vez Andalaf y Gallardo (2008), las opciones se basan en considerar que todas las empresas tienen un conjunto de posibilidades (opciones) que si son bien utilizadas incrementarán el valor de la misma. Estos autores afirman que "las opciones no son otra cosa que el reflejo del potencial de una empresa". Así el valor de una empresa es la sumatoria del valor de los negocios actuales más el potencial de una futura creación de valor. Es precisamente el valor de estas opciones el que no es capturado por las herramientas más tradicionales y esa es la causa de que la empresa quede valorada por debajo de la realidad.

A mayor redundancia y tal como señalan Amram y Kulatilaka (2000) la presencia de incertidumbre bajo un enfoque tradicional genera un menor valor del activo. El caso contrario lo representan las opciones ya que esta metodología considera que un escenario de mayor incertidumbre tiene la potencialidad de generar mayor valor del activo si es que la administración de la empresa logra identificar las opciones que le otorguen la flexibilidad necesaria para hacer frente a los vaivenes de los proyectos (Figura 1). Así la metodología basada en opciones surge como complemento del VAN.

Es interesante hacer notar como las acciones ordinarias de una compañía pueden asemejarse a las opciones. Para ello Mascareñas (2014) hace el siguiente análisis: la propiedad de una sociedad recae en los accionistas. No obstante cuando esta está endeudada se debe enfocar en saldar ese compromiso y sólo después de eso repartir lo sobrante entre los accionistas.

Si $V>D \rightarrow E=V-D \quad($ Ecu 1)

Donde $\mathrm{V}$ representa el valor de la empresa, D la magnitud de la deuda y $\mathrm{E}$ el valor de la acción Si no fuera posible saldar la deuda, la empresa debe responder incluso con su activo lo que implica que mientras no se responda a los acreedores, estos son dueños de ese activo. En este caso se tiene que:

$$
\text { Si } V \leq D \rightarrow E=0 \quad \text { (Ecu 2) }
$$

En forma análoga se puede hacer el análisis con las opciones. Así una opción de compra C sobre un activo subyacente $S$, con un precio de ejercicio $X$ se puede representar de la siguiente forma:

$$
\begin{aligned}
& \text { Si } S>X \rightarrow C=S-X \quad \text { (Eси } 3) \\
& \text { Si } S \leq X \rightarrow C=0 \quad \text { (Еси 4) }
\end{aligned}
$$

La segunda expresión nos indica que si el valor del activo subyacente es menor al precio de equilibrio acordado previamente, la opción de compra no se ejercerá. Luego es posible asimilar, financieramente hablando, las acciones ordinarias a opciones de compra sobre los activos de la compañía.

La valoración de una empresa o de un proyecto que proporciona algún tipo de flexibilidad futura opciones reales- no puede realizarse correctamente con las técnicas tradicionales de actualización de flujos futuros (VAN o TIR). Una opción real está presente en un proyecto de inversión cuando existe alguna posibilidad futura de actuación al conocerse la resolución de alguna incertidumbre actual (Fernández, 2001).

El término de opciones reales fue establecido por Stewar Myers en 1977, para hacer referencia a la aplicación de la teoría de opciones en la valoración de bienes no financieros, específicamente a la inversión en activos reales que presentaran un componente de flexibilidad, tal como la inversión en investigación y desarrollo y en la expansión de plantas de manufactura (Myers, 1977).

Las opciones reales son un método para valorar proyectos de inversión que parte de la premisa de que los proyectos de inversión reales pueden asemejarse a las opciones financieras (call y put) y no a una cartera de bonos sin riesgo como el VPN, el cual deja de ser útil cuando se presentan situaciones en las que no necesariamente el proyecto tiene que realizarse inmediatamente, es decir, cumplirse más adelante o por partes (crecimiento contingente). Dixit y Pindyck, (1994). En otras palabras, el enfoque de las opciones reales es la extensión de la Teoría de Opciones Financieras a opciones en activos reales (no financieros) que permiten modificar un proyecto con la intención de incrementar su valor. 
Las opciones reales permiten añadir valor a la empresa, al aumentar las ganancias o reducir las pérdidas. A menudo no se utiliza el término opción para describir estas oportunidades, más bien se hace referencia a ellas como intangibles más que como opciones de compra o de venta, pero cuando se evalúan propuestas de inversión importantes, estas opciones intangibles son a menudo la clave de las decisiones.

\subsection{Breve descripción de la valoración de opciones}

\subsubsection{Modelo Binomial}

Propuesto por Cox, Ross y Rubinstein en 1979. Es un modelo discreto que considera que la evolución del precio del activo subyacente varía según el proceso binomial multiplicativo; es decir, sólo puede tomar dos valores posibles, uno al alza y otro a la baja, con probabilidades asociadas $p$ y 1-p. De esta forma, al extender esta distribución de probabilidades a lo largo de un número determinado de períodos se consigue determinar el valor teórico de una opción.

\subsubsection{Modelo de Black \& Scholes ${ }^{1}$}

Los supuestos básicos del modelo de BlackScholes, al modelo binomial, tales como: mercado financiero perfecto, no existen comisiones ni costos de transacción ni de información, ausencia de impuestos, y si existen, gravarían por igual a todos los inversores, la acción o activo subyacente no paga dividendos ni cualquier otro tipo de reparto de beneficios durante el período considerado, la opción es de tipo europeo, sólo puede ejercerse a su expiración, son posibles las "ventas al descubierto" del activo subyacente, es decir, ventas sin poseer el activo, la negociación en los mercados es continua y el precio del subyacente (S) realiza un recorrido aleatorio con varianza $(\sigma 2)$ proporcional al cuadrado de dicho precio.

Valor opción call

$$
\begin{aligned}
& C_{0}=S \times N\left(d_{i}\right)-E \times N\left(d_{z}\right) e^{-r_{f} T} \\
& d_{i}=\frac{\ln (S / E)+\left(r_{f}+.5 \sigma^{2}\right) T}{\sigma \sqrt{T}}, \quad d_{z}=d_{i}-\sigma \sqrt{T}
\end{aligned}
$$

Valor opción put

$$
P_{o}=E \times N\left(-d_{z}\right) e^{-r_{f} T}-S x N\left(-d_{i}\right)
$$

\subsubsection{Simulación de Montecarlo}

Los problemas relacionados con el activo subyacente son afrontados cada vez en mayor medida, mediante simulación de Montecarlo del Valor Presente del Proyecto. La simulación Montecarlo es una técnica que implica la selección aleatoria de un resultado para cada variable de interés. Mediante la combinación de estos resultados con cantidades fijas y su respectivo cómputo, se obtiene una corrida en términos de la respuesta deseada. Esto se hace repetidamente hasta conseguir las corridas suficientes para lograr una aproximación cercana a la media, la varianza y la forma de la distribución. La simulación de Montecarlo se ha venido aplicando a una infinidad de ámbitos como alternativa a los modelos matemáticos exactos o incluso como único medio de estimar soluciones para problemas complejos. Así, en la actualidad es posible encontrar modelos que hacen uso de simulación Montecarlo en las áreas informática, empresarial, económica, industrial e incluso social.

Según Zapata, Piñeros y Castaño (2004), este es el método de análisis más versátil dado que permite utilizar cualquier distribución para modelar los tiempos para salida y restauración de los componentes, permite resolver sistemas en los cuales no existe una solución analítica, permite obtener las distribuciones de probabilidad de los índices de confiabilidad de los puntos de carga, lo cual es muy útil para valorar el riesgo de que ocurran diferentes valores de los índices.

Según García y Romero (2009), el método de simulación para resolver problemas de opciones reales es similar a la técnica que se basa en la simulación de miles de trayectorias que el valor del activo subyacente puede tomar durante la vida de la opción, dados los límites del cono de incertidumbre definido por la volatilidad del valor del activo. Esta trayectoria es presentada en la siguiente Figura 2.

\footnotetext{
1 Black, F., \& Scholes, M. (1973). The pricing of options and corporate
} liabilities. The journal of political economy, 637-654. 
Figura 2. Simulación de Montecarlo y cono de incertidumbre.

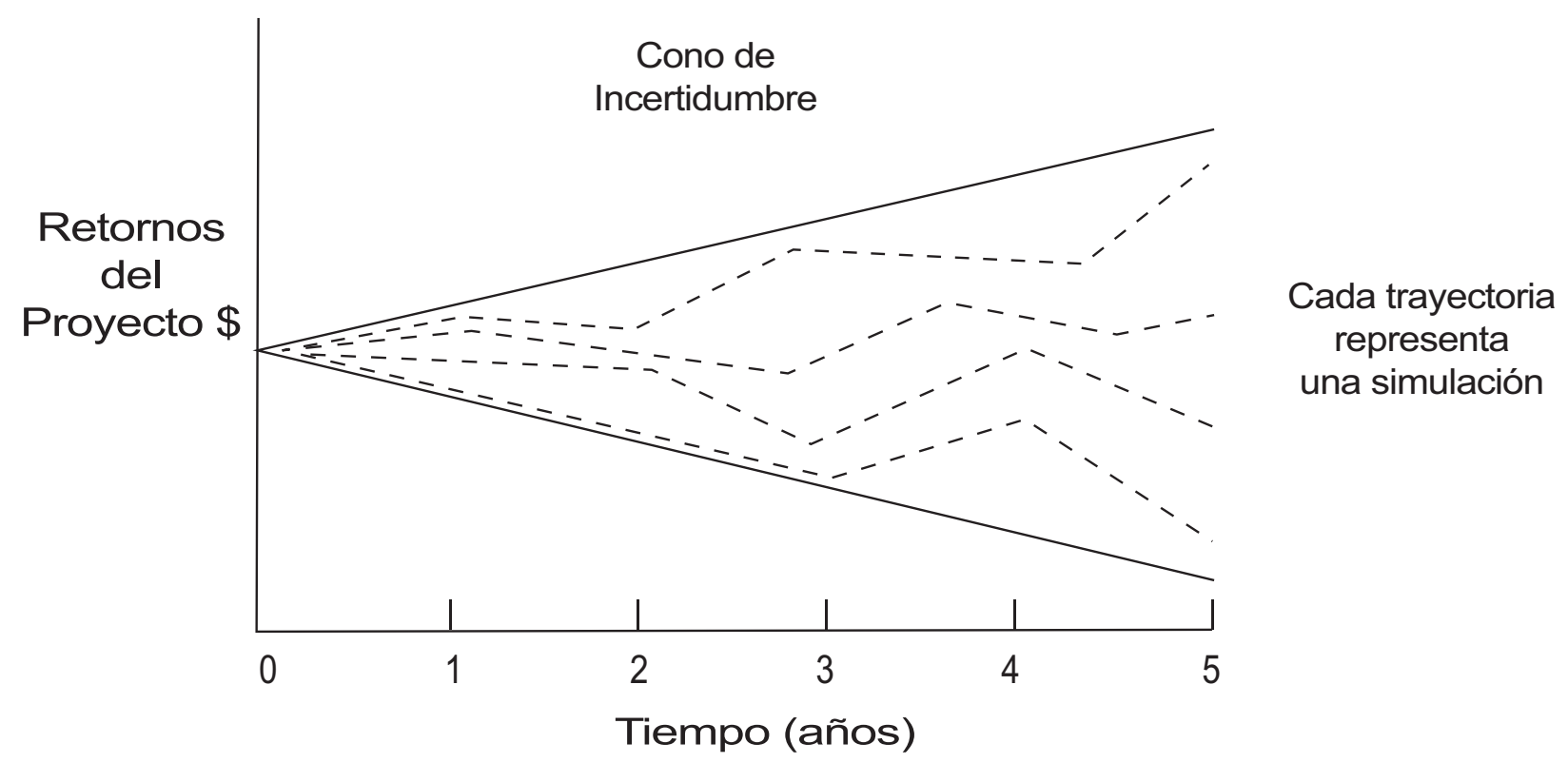

Parámetros de entrada requeridos para realizar la simulación:

- Valor Actual del Activo Sudyacente

$\left(S_{0}\right)$

- Volatilidad del Valor del Activo

(б)

- Precio de Ejercicio (X)

- Vida de la Opción (T)

Fuente: García y Romero (2009). Figura publicado con la autorización de los autores.

En el caso de una opción "call" simple, si el precio de ejercicio es menor que el valor del activo, la opción de invertir, presumiblemente será ejercido y el valor del proyecto será igual a la diferencia entre el valor del activo y el precio de ejercicio. Por el contrario, si el precio de ejercicio es mayor, el valor del activo del proyecto será igual a cero, porque la opción no será ejercida.

$$
S_{T}=S_{T-1}+S_{T-1}(r \delta t+\sigma \varepsilon \sqrt{\delta t})
$$

Donde:

$S_{T}$ y $S_{T-1}=$ valor del activo subyacente en el periodo t y t-1

$\sigma=$ volatilidad del valor del activo subyacente

$\mathcal{E}=$ valor simulado obtenido de una distribución normal estandar con media cero y varianza uno 
La simulación puede ser usada fácilmente para opciones europeas, donde hay una fecha de ejercicio fija, la vida de la opción puede ser dividida en un número determinado de períodos y simular la evolución del valor del activo para cada período. Mientras mayor sea el número de períodos, mayor es el número de simulaciones, más preciso será el resultado. Con una opción americana, debido a que ésta puede ser ejercida en cualquier momento, la simulación debe ser diseñada ajustando la vida de la opción de forma que coincida con cada fecha de ejercicio posible, lo cual es una tarea enorme. Esto hace que sea muy complejo aplicar la simulación sobre opciones secuenciales, porque cada decisión posible lleva a una nueva trayectoria, y la simulación deberá ser ajustada para cada nueva trayectoria. En la actualidad existe una gran cantidad de metodologías diseñadas para evaluar opciones mediante la simulación. Estas metodologías de simulación de opciones americanas han sido empleadas en la evaluación de opciones reales, destacando los trabajos de Cortázar \& Schwartz (1998), Cortázar (2001). Sin embargo, su implementación presenta desventajas en la obtención de ciertos indicadores como la política óptima de ejercicio y su tiempo de ejecución puede ser extenso en algunos problemas de opciones reales (Urzúa, 2004).

\section{APLICACIÓN DE LA METODOLOGÍA}

\subsection{La empresa}

Aguas Andinas S.A. es la principal empresa sanitaria del país y una de las mayores de América Latina. Provee servicios a más de 6,8 millones de habitantes en las regiones Metropolitana, De Los Ríos y De Los Lagos con altos niveles de cobertura y eficiencia, por encima del promedio de la industria. Constituye un monopolio natural regulado bajo un marco regulatorio estable, conocido y probado.

La empresa presenta una situación favorable en términos de riesgo debido principalmente a las características particulares del sector sanitario. El negocio es estacional y los resultados de la explotación pueden variar de un trimestre a otro. Los mayores niveles de demanda e ingresos se registran durante los meses de verano debido principalmente a las necesidades adicionales de agua que generan los sistemas de irrigación y otros usos externos de agua.

\subsection{Proyecciones de flujos de caja}

\subsubsection{Ingresos}

Como se enunció en la sección anterior, el número de clientes es una variable de máxima importancia en el volumen de ventas y por ende en el ingreso de Aguas Andinas. Debido a esto, es prioritario contar con los datos a futuro de este factor. Se espera que el natural crecimiento de la población, según datos extraídos de un estudio de población realizado por el instituto nacional de estadísticas (INE), en conjunto con la División de Población de la CEPAL fijan para el año 2030 una población cercana a los 8 millones de habitantes para la Región Metropolitana (Véase la Tabla 1 en donde se presenta los datos de clientes versus ingresos hasta el 2013).

Tabla 1. Clientes v/s ingresos

\begin{tabular}{|c|c|c|}
\hline & $\begin{array}{c}\text { Total Clientes } \\
\text { AP }\end{array}$ & $\begin{array}{c}\text { Ingresos } \\
\text { Operacionales } \\
\text { (MM\$) }\end{array}$ \\
\hline 2007 & 1.597 .537 & 276.340 \\
\hline 2008 & 1.828 .328 & 305.534 \\
\hline 2009 & 1.871 .287 & 328.118 \\
\hline 2010 & 1.908 .816 & 328.964 \\
\hline 2011 & 1.946 .186 & 362.768 \\
\hline 2012 & 1.984 .132 & 382.886 \\
\hline 2013 & 2.039 .298 & 402.624 \\
\hline
\end{tabular}

Fuente: Instituto Nacional de Estadísticas de Chile (INE).

\subsubsection{Costos y gastos de operación}

Para el ítem de costos y gastos procedemos de forma similar aplicando una prueba de correlación que nos ayude a determinar qué relación encontramos entre costos e ingresos (Véase la Tabla 2).

Tabla 2. Ingresos versus costos

\begin{tabular}{|c||c|c|}
\hline & $\begin{array}{c}\text { Ingresos } \\
\text { Operacionales } \\
\text { (MM\$) }\end{array}$ & $\begin{array}{c}\text { Costos } \\
\text { Operación } \\
\text { (MM\$̦) }\end{array}$ \\
\hline 2007 & 276.340 & 144.230 \\
\hline 2008 & 305.534 & 157.459 \\
\hline 2009 & 328.118 & 173.088 \\
\hline 2010 & 328.964 & 179.179 \\
\hline 2011 & 362.768 & 189.537 \\
\hline 2012 & 382.886 & 196.173 \\
\hline
\end{tabular}

Fuente: Estados financieros Aguas Andinas S.A . Información pública, disponible para todo el mercado. 


\subsubsection{Impuestos}

Del Estado de Resultados 2007 - 2013 se obtiene que el promedio de los montos en impuestos efectivamente pagados por la empresa (como porcentaje de la ganancia antes de impuesto) es de un $18,56 \%$. Los valores anuales son los siguientes (Véase la Tabla 3):

Tabla 3. Porcentaje de impuesto efectivamente pagado

\begin{tabular}{|r|c|c|c|c|c|c|c|}
\hline $\begin{array}{r}\text { Impto pagado (\% } \\
\text { efectivo) }\end{array}$ & 31 -dic-07 & 31 -dic-08 & 31 -dic-09 & 31 -dic-10 & 31 -dic-11 & 31 -dic-12 & 31 -dic-13 \\
\hline Promedio & $18,56 \%$ & $19,05 \%$ & $17,12 \%$ & $15,52 \%$ & $18,63 \%$ & $22,72 \%$ & $19,67 \%$ \\
\hline & & & & & &
\end{tabular}

Fuente: Estados financieros Aguas Andinas S.A . Información pública, disponible para todo el mercado.

\subsection{Valoración de de flujos de caja descontados (DCF)}

\subsubsection{Coeficiente Beta ${ }^{1}$}

Del modelo propuesto por el Profesor William Sharpe (1964) para estimar el precio de un activo de capital (una acción por ejemplo) a través de su propio riesgo se deriva que existe un único factor de riesgo a tomar en cuenta en la valuación de activos de capital: el riesgo sistemático, medido a través del parámetro "beta".

$$
\begin{aligned}
\mathrm{Kp}=\mathrm{Rf} & +(\mathrm{E}(\mathrm{Rm})-\mathrm{Rf})^{\star} \beta p . c d \quad(E c u \text { 6) } \\
\mathrm{Kp} & =\text { Rentabilidad exigida por el accionista } \\
\mathrm{Rf} & =\text { Tasa libre de riesgo } \\
(\mathrm{E}(\mathrm{Rm})-\mathrm{Rf}) & =\text { Premio por riesgo de mercado } \\
\beta p . c d \quad & =\text { Beta del patrimonio apalancado }
\end{aligned}
$$

Para proceder al cálculo se utilizó la fórmula (27) y los datos de rentabilidad del IPSA y de la acción de Aguas Andinas obteniendo como resultado un $\boldsymbol{\beta}=\mathbf{0 , 4 4 9 8 8}$. Esta metodología es la misma usada por la Bolsa de Comercio de Santiago.

\subsubsection{Cálculo valor empresa por flujo de caja descontado (DCF)}

Para proceder a la valoración de la empresa 4 se utilizó la expresión siguiente:

$$
V E_{c d}=\sum_{i=1}^{n} \frac{I O N_{i}(1-t)}{(1+k c)^{i}}+\sum \frac{I_{i}(1-t)}{(k c-g)^{i}}
$$

(Ecu 7)

$$
\begin{array}{ll}
\text { VEcd } & =\text { Valor Empresa con deuda } \\
\text { ION } & =\text { Ingreso operacional neto } \\
\mathrm{t} & =\text { Tasa de impuesto } \\
\mathrm{Kc} & =\text { Costo de capital de la empresa } \\
\mathrm{g} & =\text { tasa de crecimiento permanente }
\end{array}
$$

Tabla 4. Valoración empresa Flujos de caja descontados (DCF)

\begin{tabular}{|c|c|}
\hline Tasa de descuento & $7,254 \%$ \\
\hline Total Activos en $\$$ & 3.697 .725 .000 .000 \\
\hline Total deuda en $\$$ & 900.042 .114 .000 \\
\hline Total patrimonio en $\$$ & 2.797 .676 .460 .40 \\
\hline $\mathrm{N}^{\circ}$ de acciones & 6.118 .965 .160 \\
\hline Precio por acción & 457,21 \\
\hline
\end{tabular}

Fuente: Elaboración propia.

\subsection{Valoracion de la empresa mediante opciones reales}

En el inicio del artículo se mencionó que valorar una empresa es un desafío mayor en el entendido que se debe considerar innumerables variables y condiciones que pueden derivar en resultados diferentes según sea la óptica y los supuestos considerados de quien realiza la evaluación. Concepto clave además es la aparición de la incertidumbre que puede afectar el rendimiento esperado de una inversión tanto a la baja como al alza. Esta es una característica fundamental en la metodología de las Opciones ya que se consideran ambas posibilidades a diferencia de los enfoques más tradicionales en que prima el punto de vista del menor rendimiento. 
A continuación se mostrará la valoración de Aguas Andinas a través de Opciones usando los métodos Binomial, de Black-Scholes y Montecarlo.

\subsubsection{Riesgo medido a través de la varianza}

Preliminar al desarrollo de estos tres métodos, es necesario realizar el cálculo de la variabilidad de los activos. Tomando como fuente los datos de transacciones en bolsa de la acción de Aguas Andinas se obtuvo el valor de la varianza histórica. El paso siguiente es el cálculo de la varianza que se usará para la valoración de la empresa a través de Opciones. Esto se logra incorporando el riesgo a los flujos de caja mediante el movimiento geométrico browniano².

Tabla 4. Valoración empresa Flujos de caja desconectados (DCF)

\begin{tabular}{|cc}
\hline Tasa de descuento & $\mathbf{7 , 2 5 4 \%}$ \\
\hline Total Activos en \$ & 3.697 .725 .000 .000 \\
\hline Total deuda en \$ & 900.042 .114 .000 \\
Total patrimonio en $\$$ & 2.797 .676 .460 .407 \\
\hline No de acciones & 6.118 .965 .160 \\
\hline Precio por acción & 457,21 \\
\hline
\end{tabular}

En donde:

VTAS $_{t+1}=$ Ingresos por ventas periodo $t+1$

VTAS $_{t}=$ Ingresos por ventas periodo $t$

$\mu \quad=$ Tasa de crecimiento promedio

$\delta^{2}=$ Varianza

$\delta=$ desviación estándar
$\mathrm{T}=$ periodo de tiempo

$Z$ = ruido añeatorio que se distribuye normalmente, $\operatorname{con} \mu=o$ y $\operatorname{con} \delta=1$

\subsubsection{Deuda financiera de la empresa}

A través de un análisis a la estructura de los pasivos de Aguas Andinas S.A. es posible obtener los indicadores de tasa y plazo de la deuda con los cuales más adelante se valora la empresa por medio de opciones reales (Véase las Tablas 5 y 6 ).

\subsubsection{Valoración empresa modelo binomial}

La Figura 4 muestra el resumen del modelo binomial

\subsubsection{Valoración empresa modelo Black \& Scholes}

Para este método se recurre a la fórmula siguiente:

$$
C=S N\left(d_{1}\right)-E e^{r f t} N\left(d_{2}\right)
$$

Con esto los resultados obtenidos se muestran en la tabla 7

Tabla 7. Resultados de la Valoración

\begin{tabular}{|l|r|}
\hline$d 1$ & 1,825 \\
\hline$d 2$ & 0,698 \\
\hline$N(d 1)$ & 0,966 \\
\hline$N(d 2)$ & 0,757 \\
\hline $\mathrm{Ce}$ & 2.896 .614 .470 .185 \\
\hline
\end{tabular}

\begin{tabular}{|l|l|}
\hline Precio de la acción & 473,38 \\
\hline
\end{tabular}

Fuente: Elaboración propia.

Figura 3. Cálculo de la varianza del activo, mediante movimiento geométrico Browniano.

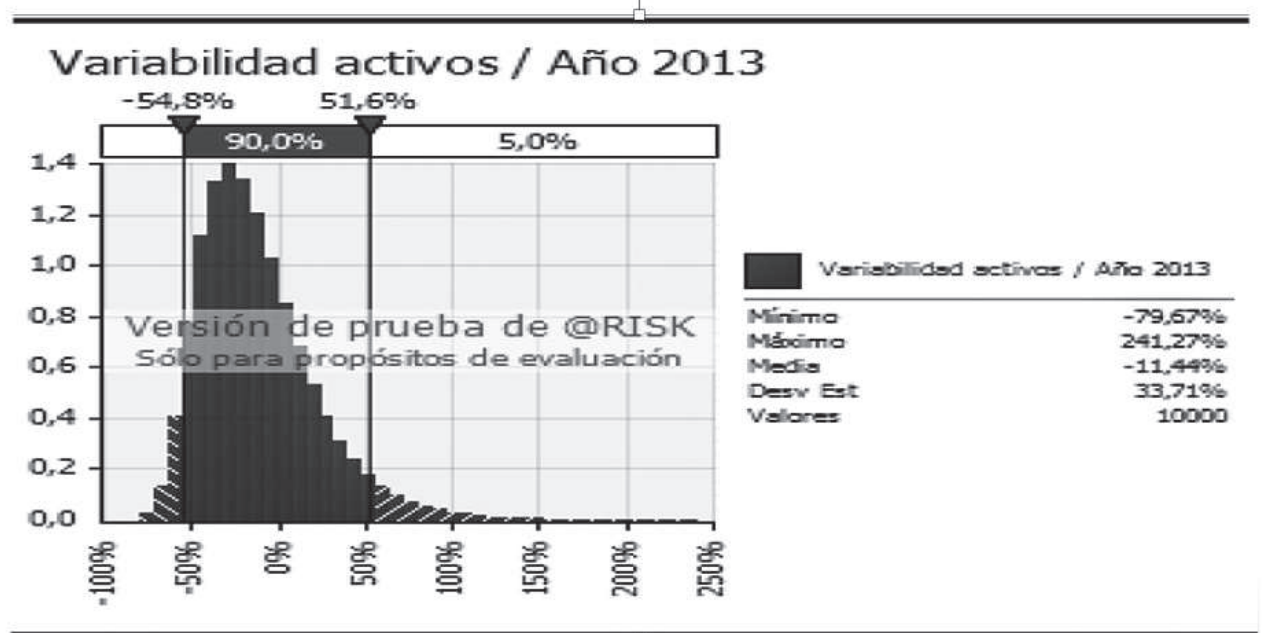

Fuente: Elaboración propia.

2 El movimiento browniano es el movimiento aleatorio que se observa en algunas partículas microscópicas que se hallan en un medio fluido (por ejemplo, polen en una gota de agua). Recibe su nombre en honor al escocés Robert Brown, biólogo y botánico que descubrió este fenómeno en 1827 y observó que pequeñas partículas de polen se desplazaban en movimientos aleatorios sin razón aparente. 
Tabla 5. Deuda consolidada empresa Aguas Andinas S.A.

\begin{tabular}{|c|c|c|c|c|c|c|c|c|c|c|c|c|c|c|}
\hline \multicolumn{15}{|c|}{ ESTRUCTURA DE PASIVOS FINANCIEROS (MMS) } \\
\hline & \multicolumn{2}{|c|}{ CORRIENTE } & \multicolumn{11}{|c|}{ NO CORRIENTE } & \multirow{3}{*}{ Tasa } \\
\hline & 9od & 91-365d & $13 m-3 a$ & $3 a-5 a$ & $a b r-19$ & dic-26 & feb-28 & jun-28 & $a b r-31$ & jun-32 & oct-33 & $a b r-35$ & $a b r-36$ & \\
\hline MESES & 3 & 12 & 36 & 60 & 64 & 156 & 170 & 174 & 208 & 222 & 238 & 256 & 268 & \\
\hline \multirow{4}{*}{$\begin{array}{l}\text { Préstamos } \\
\text { Bancarios }\end{array}$} & 1.461 & 1.147 & 13.094 & 0 & 0 & 0 & 0 & 0 & 0 & 0 & 0 & 0 & 0 & $6,04 \%$ \\
\hline & 648 & 371 & 2.770 & 6.392 & 0 & 0 & 0 & 0 & 0 & 0 & 0 & 0 & 0 & $6,07 \%$ \\
\hline & 189 & 0 & 9.535 & 0 & 0 & 0 & 0 & 0 & 0 & 0 & 0 & 0 & 0 & $5,91 \%$ \\
\hline & 2.736 & 1.567 & 11.693 & 26.983 & 0 & 0 & 0 & 0 & 0 & 0 & 0 & 0 & 0 & $6,07 \%$ \\
\hline \multirow{7}{*}{ AFR } & 0 & 2.023 & 0 & 0 & 0 & 0 & 0 & 0 & 0 & 0 & 0 & 0 & 0 & $2,67 \%$ \\
\hline & 0 & 2.175 & 0 & 0 & 0 & 0 & 0 & 0 & 0 & 0 & 0 & 0 & 0 & $9,34 \%$ \\
\hline & 0 & 236 & 0 & 0 & 0 & 0 & 0 & 0 & 0 & 0 & 0 & 0 & 0 & $2,85 \%$ \\
\hline & 0 & 0 & 0 & 0 & 0 & 0 & 0 & 89.233 & 0 & 0 & 0 & 0 & 0 & $3,66 \%$ \\
\hline & 0 & 0 & 0 & 0 & 0 & 0 & 0 & 21.981 & 0 & 0 & 0 & 0 & 0 & $4,29 \%$ \\
\hline & 0 & 0 & 0 & 0 & 0 & 0 & 8.707 & 0 & 0 & 0 & 0 & 0 & 0 & $3,65 \%$ \\
\hline & 0 & 0 & 0 & 0 & 0 & 0 & 0 & 5.150 & 0 & 0 & 0 & 0 & 0 & $3,76 \%$ \\
\hline \multirow{13}{*}{ BONOS } & 273 & 5.833 & 11.945 & 11.945 & 0 & 47.778 & 0 & 0 & 0 & 0 & 0 & 0 & 0 & $4,68 \%$ \\
\hline & 434 & 58.221 & 0 & 0 & 0 & 0 & 0 & 0 & 0 & 0 & 0 & 0 & 0 & $3,39 \%$ \\
\hline & 74 & 7.391 & 16.747 & 0 & 0 & 0 & 0 & 0 & 0 & 0 & 0 & 0 & 0 & $4,03 \%$ \\
\hline & 46 & 0 & 0 & 23.157 & 0 & 0 & 0 & 0 & 0 & 0 & 0 & 0 & 0 & $4,17 \%$ \\
\hline & 114 & 3.706 & 12.097 & 0 & 0 & 0 & 0 & 0 & 0 & 0 & 0 & 0 & 0 & $3,08 \%$ \\
\hline & 435 & 0 & 0 & 0 & 0 & 0 & 0 & 0 & 41.058 & 0 & 0 & 0 & 0 & $4,14 \%$ \\
\hline & 187 & 0 & 29.061 & 0 & 0 & 0 & 0 & 0 & 0 & 0 & 0 & 0 & 0 & $3,33 \%$ \\
\hline & 342 & 0 & 0 & 0 & 0 & 0 & 0 & 0 & 0 & 0 & 35.189 & 0 & 0 & $3,81 \%$ \\
\hline & 138 & 0 & 0 & 0 & 0 & 0 & 0 & 0 & 0 & 38.747 & 0 & 0 & 0 & $3,94 \%$ \\
\hline & 142 & 0 & 10.284 & 10.284 & 2.571 & 0 & 0 & 0 & 0 & 0 & 0 & 0 & 0 & $3,62 \%$ \\
\hline & 511 & 0 & 0 & 0 & 0 & 0 & 0 & 0 & 0 & 0 & 0 & 53.412 & 0 & $3,93 \%$ \\
\hline & 436 & 0 & 0 & 0 & 0 & 0 & 0 & 0 & 0 & 0 & 0 & 0 & 46.524 & $3,81 \%$ \\
\hline & 193 & 2.589 & 5.916 & 5.916 & 0 & 0 & 0 & 28.102 & 0 & 0 & 0 & 0 & 0 & $6,63 \%$ \\
\hline
\end{tabular}

Fuente: Estados financieros publicados por Aguas Andinas en la Superintendencia de Valores y Seguros de Chile (2013).

Tabla 6. Duración de la deuda de empresa Aguas Andinas

\begin{tabular}{|c|c|c|c|c|c|c|c|c|c|c|c|c|c|c|}
\hline \multicolumn{15}{|c|}{ ESTRUCTURA DE PASIVOS FINANCIEROS (MMSS) } \\
\hline \multirow[b]{3}{*}{ MESES } & \multicolumn{2}{|c|}{ CORRIENTE } & \multicolumn{11}{|c|}{ NO CORRIENTE } & \multirow{3}{*}{ TOTAL } \\
\hline & $90 \mathrm{~d}$ & 91-365d & $13 m-3 a$ & $3 a .5 a$ & abr-19 & dic -26 & feb-28 & jun-28 & $a b r-31$ & jun. 32 & oct-33 & abr 35 & $3 b r=36$ & \\
\hline & 3 & 12 & 36 & 60 & 64 & 156 & 170 & 174 & 208 & 222 & 238 & 256 & 268 & \\
\hline DEUDA (MMS) & 8.360 & 85.260 & 123.142 & 84.678 & 2.571 & 47.778 & 8.707 & 144.466 & 41.058 & 38.747 & 35.189 & 53.412 & 46.524 & 719.892 \\
\hline Peso Relativo & 0,011613 & 0,118434 & 0,171056 & 0,117626 & 0,003571 & 0,066369 & 0,012095 & 0,200677 & 0,057034 & 0,053824 & 0,04888 & 0,074194 & 0,064626 & \\
\hline Meses prop. & 0,03484 & 1,421212 & 6,15802 & 7,057539 & 0,228571 & 10,35349 & 2,056188 & 34,91788 & 11,86303 & 11,9489 & 11,63351 & 18,99377 & 17,31968 & 133,987 \\
\hline
\end{tabular}

Fuente: Estados financieros publicados por Aguas Andinas en la Superintendencia de Valores y Seguros de Chile (2013).

Figura 4. Resumen modelo binomial con 90 iteraciones.

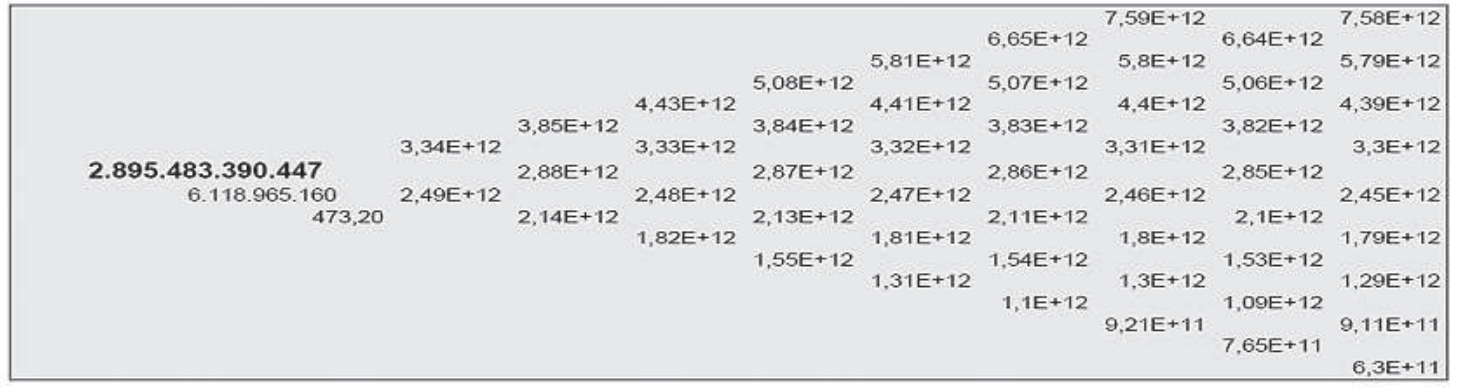

Fuente: Elaboración propia. 


\subsubsection{Valoración empresa simulación de} Montecarlo

Para esta simulación se usó la fórmula:

$$
x t=x q * e^{\left(\left(\mu \frac{\sigma^{2}}{2}\right) T+\sigma * Z * \sqrt{T}\right)}
$$

(Ecu 9) Modelación del valor de los activos con 10.000 iteraciones
Los resultados se muestran en la figura 5 y en la tabla 8 .

\subsubsection{Resumen de las valoraciones}

A continuación se presente un resumen de los resultados entregados por la valoración tradicional de los flujos de caja descontados y por la valoración por medio de la teoría de opciones. (Ver Tabla 7 y Figura 6)

Figura 5. Valor empresa simulación de Montecarlo.

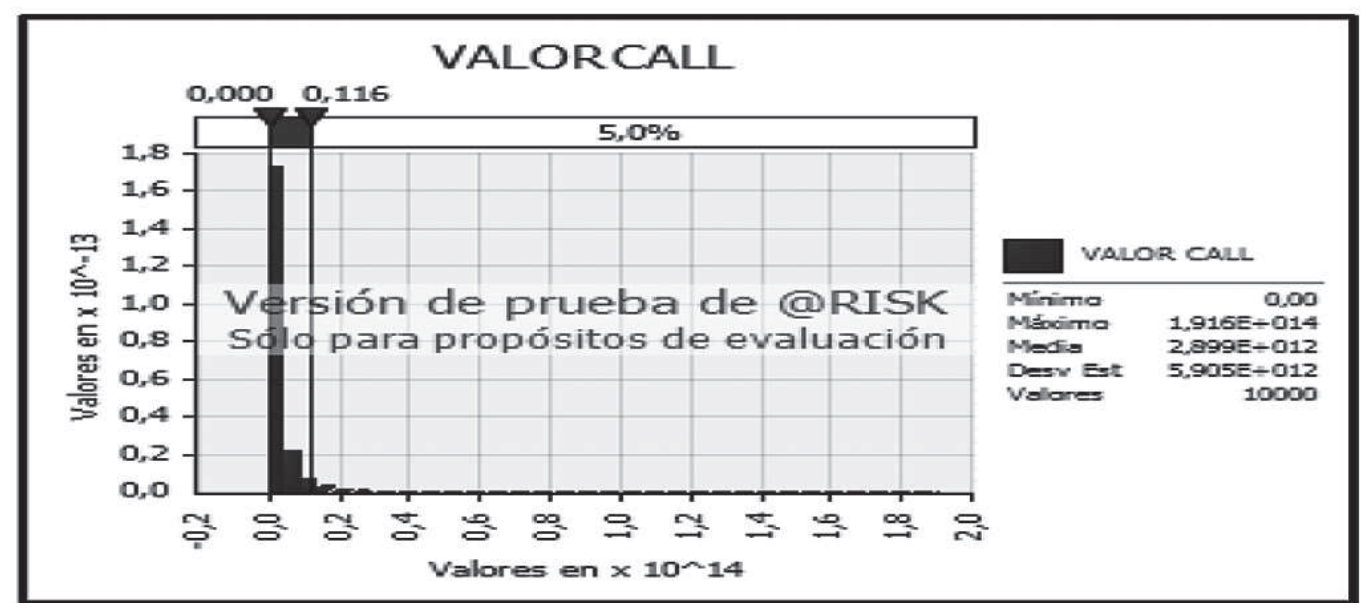

Fuente: Elaboración propia en base a programa @ Risk

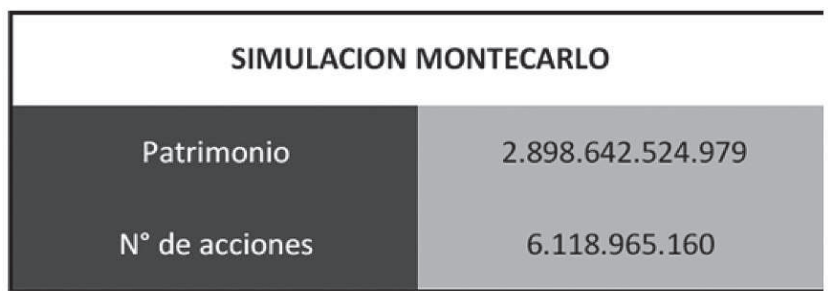

Precio de la acción

473,71

Fuente: Elaboración propia.

Tabla 7. Resumen de los valoraciones de la empresa

\begin{tabular}{|c|cc|}
\hline \multicolumn{1}{c}{ ENFOQUE } & METODO & PRECIO POR ACCION \\
\hline TRADICIONAL & $\begin{array}{r}\text { FLUJO DE CAJA DESCONTADO } \\
\text { (DCF) }\end{array}$ & 457,21 \\
& BINOMIAL & 473,2
\end{tabular}

Fuente: Elaboración propia. 
Figura 6. Precio de cierre de las acciones de Aguas Andinas S.

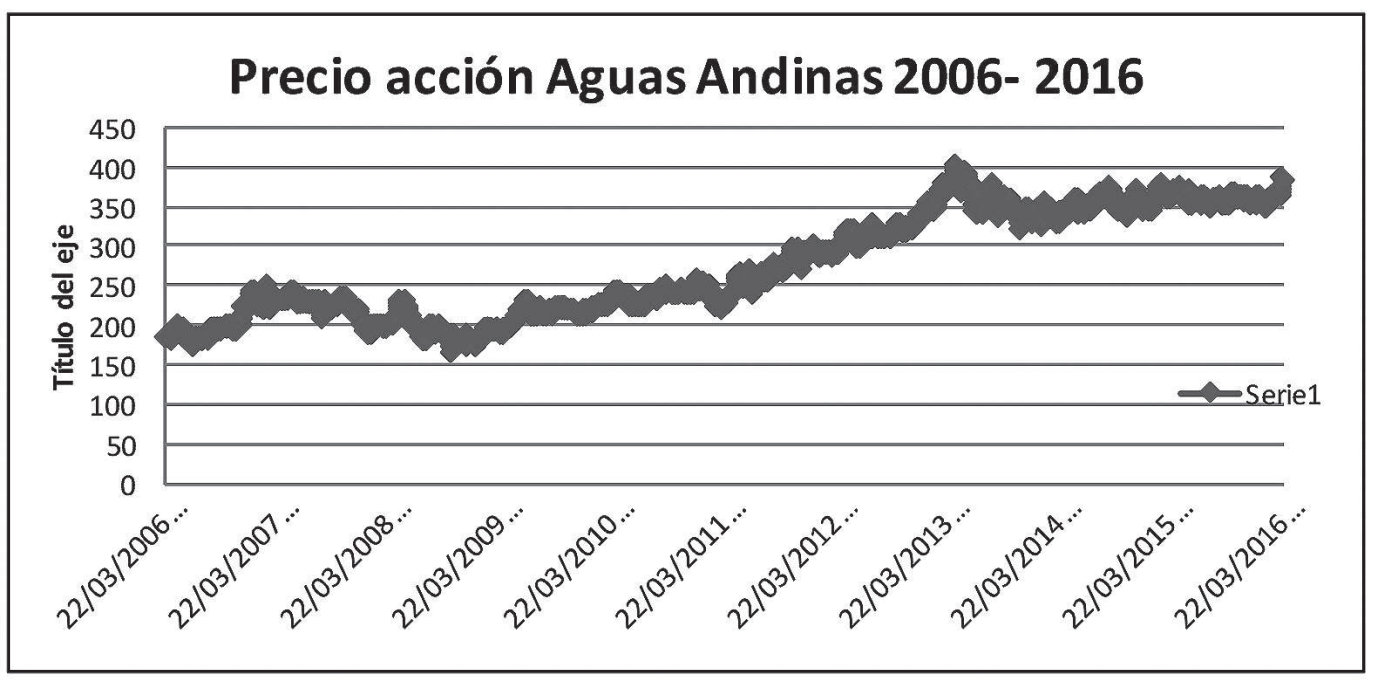

Fuente: Bolsa de Comercio de Santiago de Chile.

\section{RESULTADOS}

La valoración por DCF entregó un valor de $\$ 457,21$ por acción el que comparado con último precio $\$ 383$, muestra que ésta acción se encontraría subvalorada. La valoración de las acciones a través de las metodologías como binomial, Black y Scholes y Montecarlo arrojan valores superiores a la valoración que se realizó a través de la metodología de los flujos de caja descontados. Además la metodología de opciones reales entregó resultados superiores al valor de cotización de la acción registrado en la bolsa de comercio de Santiago de Chile

Las metodologías de opciones usadas (Binomial, Black-Scholes y simulación de Montecarlo) dieron como resultado valoraciones muy similares en torno a los $\$ 473$ por acción. El principal factor que influye en el ritmo de los ingresos de este tipo de compañías viene dado por el crecimiento en la masa de clientes. En ese sentido, las proyecciones del mercado fijan en un 3,9\% de aumento anual promedio para los ingresos de Aguas Andinas, porcentaje muy similar al $4,08 \%$ usado en este estudio y que fue obtenido de la correlación del número de clientes e ingresos operacionales.

En términos generales, empresas como Aguas Andinas muestran perfiles de ingresos relativamente seguros siendo las regulaciones del fiscalizador $y$ las condiciones climáticas las principales fuentes de riesgo.

\section{CONCLUSIONES}

Valorar las acciones mediante opciones reales es todo un desafío, ya que debemos modelar el comportamiento de los flujos operaciones de la empresa de igual manera que un activo financiero. Un elemento clave es la determinación de la variabilidad de los flujos futuros, en condiciones de incertidumbre, recurriendo habitualmente al movimiento geométrico browniano para determinar la varianza.

El hecho de que los precios por acción superen los mostrados en las transacciones de la Bolsa de Comercio se corresponde con las expectativas que los diversos agentes tienen sobre el futuro de Aguas Andinas. Una rápida revisión de algunos informes del mercado accionario revela confianza en estos papeles basándose en el plan de inversiones de la compañía y en las proyecciones en el negocio de distribución y tratamiento de aguas.

A la luz de los resultados, es posible inferir que la metodología de opciones para la valoración de empresas genera un valor adicional, debido principalmente al impacto positivo que tiene el riesgo sobre el valor de la acción y a la variabilidad de los ingresos que pueden darse en distintos escenarios.

Considerando la diferencia en el valor de las acciones obtenidos a través de la metodología DCF y la de Opciones, podría concluirse que el enfoque 
tradicional no tiene gran utilidad, sin embargo, esto no es correcto. Para suplir potenciales errores en los resultados, parece óptimo considerar métodos que incluyan en su análisis el impacto de la incertidumbre y la flexibilidad que existe en un proyecto o en la vida de una compañía.

La metodología de opciones reales, tiene poca presencia en los estudios de valoración de empresas, no obstante, las grandes potencialidades que esta ofrece. Se requiere que se siga investigando su aplicación en los más diversos ámbitos de la producción y economía, comparando sus resultados con otras técnicas de valuación.

\section{REFERENCIAS BIBLIOGRÁFICAS}

[1] Alegría, E. \& Celedón M. (2006). Historia del sector sanitario chileno: De la gestión estatal hasta el proceso de privatización. Instituto de investigación de las Naciones Unidas para el desarrollo social.

[2] Amram, M., Kulatilaka, N., \& Martha Amram, N. K. (2000). Opciones Reales: Evaluación de inversiones en un mundo incierto (No. 658.15/ A52rE).

[3] Andalaf A. \& Gallardo M. (2008). Análisis de la incorporación de flexibilidad en la evaluación de proyectos de inversión utilizando opciones reales y descuento de flujos dinámico. Horizontes Empresariales, 7(1), 41-56.

[4] Black, F., \& Scholes, M. (1973). The pricing of options and corporate liabilities. The journal of political economy, 637-654.

[5] Cortazar, G., Schwartz, E. S., \& Casassus, J. (2001). Optimal exploration investments under price and geological-technical uncertainty: a real options model. R\&D Management, 31(2), 181-189.

[6] Cortazar, G., Schwartz, E. S., \& Salinas, M. (1998). Evaluating environmental investments: A real options approach. Management Science, 44(8), 1059-1070.
[7] Cox, J. C., Ross, S. A., \& Rubinstein, M. (1979). Option pricing: A simplified approach. Journal of financial Economics, 7(3), 229-263.

[8] Dixit, A. K., \& Pindyck, R. S. (1994). Investment under uncertainty. Princeton university press.

[9] Fernández, P. (2001). Valoración de opciones reales: dificultades, problemas y errores. IESE Business School-Universidad de Navarra.

[10]Romero, R. R., \& García, F. A. (2009). Caracterización y análisis de modelos de evaluación económica de proyectos de inversión bajo incertidumbre. Revista Ingeniería Industrial, 8(1), 35-50.

[11] Gutiérrez, M. , \& González, J. (2014). Opciones reales implícitas en una empresa distribuidora de alimentos. Industrial Data, 17(2), 39-47.

[12] Markowitz, H. (1952). Portfolio selection. The journal of finance, 7(1), 77-91.

[13] Mascareñas J. (2014). Las acciones ordinarias como opciones sobre el activo de la empresa. Monografías de Juan Mascareñas sobre Finanzas Corporativas ISSN: 1988-1878

[14] Memorias anuales años 2008-2013. Aguas Andinas S.A.

[15] Myers S.C. (1977). Determinants of corporate borrowing. Journal of Financial Economic, 5(2), 147-175.

[16] Sapag N. y Sapag R. (2000), Preparación y Evaluación de Proyectos. $4^{a}$ edición. Colombia: Mc Graw Hill.

[17] Urzúa, J. L. (2004).Valorización de Opciones Reales Multidimensionales Mediante Simulación de Monte Carlo utilizando el Algoritmo LSM. Chile: Pontificia Universidad Católica de Chile.

[18] Zapata C. J., Piñeros L. C., y Castaño D. A. (2004). El método de simulación de Montecarlo en estudios de confiabilidad de sistemas de distribución de energía eléctrica. Scientia et Technica, 1(24), 55-60. 